\title{
LA SINTAXIS DE LOS ENUNCIADOS TÉTICOS Y CATEGÓRICOS EN DOS TRADUCCIONES DE CORAZONES SOLITARIOS \\ DE RUBEM FONSECA AL ESPAÑOL
}

\author{
THE SYNTAX OF THETIC AND CATEGORICAL UTTERANCES \\ IN TWO TRANSLATIONS INTO SPANISH OF BRAZILIAN \\ RUBEM FONSECA'S SHORT NOVEL CORAÇÕES SOLITÁRIOS
}

Paulo Pinheiro-Correa*

\section{RESUMEN}

En este estudio se analizan los recursos sintácticos empleados en dos traducciones distintas al español del cuento brasileño Corazones solitarios, de Rubem Fonseca. Hemos analizado los 13 casos en los que hubo discrepancia en el orden sujeto-verbo entre la primera y la segunda traducción a la luz de la pragmática de Lambrecht $(1994,2000)$ y los estudios de Sasse $(1987,2006)$ sobre oraciones téticas. Hemos clasificado los enunciados encontrados en "téticos" o "categóricos" y hemos analizado la relación entre las soluciones sintácticas empleadas por los distintos traductores y la estructura informativa de los enunciados. Los resultados muestran recursos que preservan la estructura informativa y otros que la cambian; cambios en la función sintáctica de referentes para preservar la estructura informativa del original y alteraciones en la estructura entre el original y la traducción para obtener los mismos efectos informativos logrados en el original.

Palabras-clave: traducción; literatura brasileña; estructura informativa.

\section{ABSTRACT}

In this paper syntactic resources used in two translations into Spanish of Brazilian Rubem Fonseca's short story Corações solitários ("Lonely hearts") are analyzed. We studied all 13 occurrences where discrepancies in subject-verb word order between the two translations were found. Within Lambrecht's $(1994,2000)$ Pragmatics and Sasse's $(1987,2006)$ studies on theticity the statements found were grouped in "thetic" or "categorical" and the relation between syntactic solutions offered by each translator and the information structure of the statements was considered. Results show solutions that maintain the original information structure and others that shift it; syntactic changes done in order to preserve original information structure and structural changes done in order to reach the same informative effects showed in original statements.

Keywords: translation; brazilian literature; information structure.

\footnotetext{
* Universidade Federal Fluminense (UFF), Niterói (RJ), Brasil. papicorrea@gmail.com
} 


\section{INTRODUCCIÓN. LAS FUNCIONES INFORMATIVAS Y LA TRADUCCIÓN}

En este estudio enfocaremos el tema de las funciones informativas, tales como los definen Dik (1989) y los teóricos de la pragmática Lambrecht (1994, 2000) y Sasse $(1987,2006)$ y en el ámbito del español, Gutiérrez Ordóñez (1997) y Zubizarreta (1999), entre otros, en dos traducciones del cuento Corazones solitarios, del brasileño Rubem Fonseca (1975). Los textos analizados son los siguientes: la primera traducción publicada en Sudamérica ${ }^{1}$, de Andrea Diessler, publicada en Buenos Aires, Argentina, en 1978 y la segunda, de John O’Kuinghttons, publicada en Chile en 2014.

Específicamente, trataremos en este trabajo de los enunciados téticos o de foco sentencial y las cuestiones relacionadas a su traducción. Wangi Cege (2013:17) discute los límites que la gramática de cada lengua ofrece en el momento de traducir elementos sutiles tales como las funciones pragmáticas:

Debido a las señaladas diferencias en la estructura gramatical de las lenguas fuente y meta, pueden observarse algunos cambios en el contenido informativo del mensaje. Mona Baker (1992:86) cree que algunos detalles que serían ignorados en el texto fuente pero que tienen que estar especificados en la lengua meta pueden plantearle un serio dilema al traductor, especialmente si dichos detalles no se pueden inferir razonablemente a partir del contexto. Así un aspecto puede ser considerado opcional en determinada lengua pero puede ser obligatorio en otra ${ }^{2}$.

Y después concluye: "Un traductor que tiene por objetivo que su lector acepte una traducción como un texto legítimo (...)tendrá que ajustar ciertos rasgos de la organización del texto fuente para estar al tanto de las maneras preferidas de organizar la información, las prioridades y las normas de la lengua meta (Wangi Cege 2013:18) ${ }^{3 \prime \prime}$.

En la misma línea, Hajicová y Sgall (2003:113-14) al discutir las funciones informativas en la traducción llaman la atención a la diferencia tipológica existente

1. La primera traducción de la que tenemos noticia fue realizada por Pablo del Barco en 1977, para Alfaguara, España. No está incluida en este estudio porque nos ceñimos a las traducciones realizadas en Sudamérica.

2. Traducción del autor, del original en inglés: Due to such differences in the grammatical structure of the source and target languages, certain changes in the information content of the message during the translation process may be observed. Mona Baker (1992:86) believes that details that might be ignored in the source text but which bave to be specified in the target language can pose a serious dilemma for the translator especially if they cannot be reasonably inferred from the context. Thus, an aspect may be regarded as optional in a certain language but may be obligatory in another.

3. Traducción del autor, del original en inglés: A translator whose aim is for the reader to accept a translation as a text in its own right will (...) have to adjust certain features of the source text organization to be in line with the preferred ways of organizing information, priorities and norms of the target language. 
entre las lenguas en lo que se refiere a la expresión de funciones informativas. Así, comentan el caso de lenguas cuyo orden SVO es fijo y comparan lenguas de ese tipo -como el inglés - con lenguas eslavas, en las que, según los autores, la estructura informativa determinaría libremente el orden de palabras. Los autores ubican el español en una posición intermedia entre esos dos extremos.

Hassler (2008) analiza las funciones informativas en la traducción de tramos seleccionados de la novela El Código Da Vinci a distintas lenguas europeas con el objeto de saber si "las soluciones a las lenguas son diferentes del original y si están determinadas por restricciones culturales o lingüísticas" en lo que se refiere a la traducción de secuencias oralizadas que representan diálogos (Hassler 2008:121). Y concluye que, en los diálogos que analizó, la expresión de funciones informativas como aquellas de que nos ocupamos en este estudio "no depende solamente de las decisiones del traductor, sino también de los recursos lingüísticos de la lengua" (Hassler, 2008:141).

En las próximas secciones del artículo exploraremos la relación entre estructura informativa y sintaxis: en (1) presentamos el marco teórico y los conceptos tético y categórico, fundamentales en la descripción de la estructura informativa. En (2) discutimos la metodología de obtención y tratamiento de los datos y en (3) se presentan los resultados, discutidos caso a caso. La conclusión se presenta en (4).

\section{MARCO TEÓRICO: ENUNCIADOS TÉTICOS Y CATEGÓRICOS Y EL ORDEN DE PALABRAS}

Diversos estudios en el ámbito de la Pragmática (Lambrecht 1994, 2000, Sasse $1987,2006)$ proponen una diferencia en la función informativa de los enunciados en un texto, que pueden ser categóricos o téticos. Mientras los enunciados categóricos - más comunes en las lenguas naturales - están compuestos de dos elementos informativos: tópico-comentario, o foco-presuposición, los téticos no presentan esa estructura binaria. Más bien, se presentan como una proposición única, en la que no hay un referente sobre el cual se dice algo, sino que dichos enunciados presentan un estado de cosas en el que cualquier sujeto que venga a aparecer dentro de una oración tética presentará un estatus no-relacionado a la noción de tópico.

Lambrecht (2000:624), en ese mismo sentido, propone que las lenguas presentan estrategias de destopicalización para guiar la interpretación de si un enunciado es tético o categórico:

(...) la diferencia entre las construcciones de foco en el predicado [categóricas] y las de foco en la sentencia [téticas] involucra, respectivamente, la presencia vs. ausencia de un sujeto 
topical y, por ende, de una relación tópico-comentario o de aboutness entre el referente sujeto y la proposición. Para que se pueda interpretar determinada sentencia como de articulación de foco sentencial [tética] hace falta que se marque el elemento en cuestión de manera que no se lo interprete como una expresión topical ${ }^{4}$.

Para este autor, la operación denominada destopicalización se da cuando se emplean mecanismos morfosintácticos o prosódicos para señalar que el sujeto de determinada oración es no topical. Entre esos mecanismos se encuentran el cambio de posición del sujeto en relación con el verbo (es decir, si el sujeto topical aparece en posición preverbal [SV], el sujeto no topical aparecería en posición posverbal [VS]).

Varios autores (Lambrecht 2000, Sasse 2006, Sornicola 1995) señalan para la oración tética en español el orden de palabras verbo-sujeto (a partir de ahora, VS), lo que promueve una diferencia sintáctica entre esas oraciones y las categóricas, de orden no marcado SV en esa lengua. Sin embargo, no se trata de una característica universal, pues la teticidad puede presentarse de varias maneras. Puede incluso no manifestarse sintácticamente: puede que se manifieste tan solo en la dimensión prosódica y puede que se manifieste solo informativamente, según la lengua. La distribución de los sujetos de oraciones téticas depende del grado de gramaticalización de la relación sujeto-predicado en cada lengua (SASSE 1987).

A diferencia del español, en portugués brasileño (a partir de ahora, PB), lengua del cuento original, un sujeto u otro referente desvinculado de la idea de tópico, absolutamente nuevo en el discurso o incluso un $\mathrm{SN}$ genérico puede ocupar la posición inicial de la oración, lo que asigna al enunciado una disposición VS incluso en oraciones téticas. Pinheiro-Correa (2015), en una comparación entre PB y español de Argentina, señala que no parecen operar sobre el PB las mismas restricciones del español que determinan que en esta lengua las oraciones téticas tengan orden no marcado verbo-sujeto. Kato (1989) discute la tendencia del PB a un orden más fijo SVO, independientemente de la función pragmática (tópico, foco informativo, foco contrastivo, etc.) que tenga el argumento en la oración.

Por último, y no menos importante, está la observación de Sasse (1987), de que no es el orden lo que determina la estructura informativa de una oración, pues incluso en una lengua que marque sintácticamente la diferencia entre enunciados téticos y categóricos, un enunciado, aunque tético, puede presentar una disposición sujeto-verbo por otros factores. Y puede que determinado traductor convierta en

4. Traducción del autor, del original en inglés: (...) the difference between PF and SF constructions crucially involves the presence vs. Absence of a topical subject and bence of a topic-comment, or aboutness, relation between the subject referent and the proposition. For a sentence to be interpreted as baving SF articulation it is therefore logically necessary that the relevant constituent be marked in such a way that it will not be construed as a topic expression. 
categórico un enunciado que en el original es tético. A estos problemas vamos a dedicar las próximas secciones de este artículo.

\section{METODOLOGÍA}

La metodología consiste de la compilación de un corpus paralelo que contiene las dos traducciones del cuento publicadas en español. Se analizaron en su integralidad dos traducciones del cuento Corazones Solitarios, de Rubem Fonseca. La primera, realizada por Andrea Diessler y publicada en Argentina en 1978 y la segunda, de John O’Kuinghttons, publicada en Chile.

Para el objetivo de este análisis cualitativo, era necesario considerar enunciado por enunciado en cada versión y la relación entre ellos. Con la finalidad de obtener un paralelismo semiautomático entre los enunciados de la primera y de la segunda traducción, utilizamos el software gratuito de alineamiento YouAlign (Terminotix Inc., 2009-2017), que nos permitió organizar lado a lado cada una de las oraciones de los textos, en un único archivo. El etiquetado de las funciones pragmáticas se realizó de manera manual en el archivo ya alineado.

Los referentes se constituyeron de pronombres o frases nominales plenas. Y hemos considerado las siguientes posibilidades de orden de palabras:

a) sujeto antepuesto al verbo;

b) sujeto pospuesto al verbo en posición no final y

c) sujeto pospuesto al verbo en posición final de oración.

El criterio de selección de datos, a su vez, consideró aquellos SN cuya posición presentó variación relativamente al verbo entre las dos versiones del texto, para investigar las posibilidades de orden de palabras que ofrecen las dos versiones al español para un mismo enunciado. El trabajo se orientó primordialmente al estudio de las decisiones de cada traductor en la solución de este problema de interacción entre sintaxis y pragmática, es decir, cómo se resolvió en cada traducción al español la expresión o no de la teticidad manifestada en la sintaxis. El estudio ha tenido como punto de partida la traducción de 1978. La identificación de la estructura informativa de los datos del original, cuya sintaxis es opaca en la lengua original, se vio facilitada por el análisis contrastivo con sus traducciones al español. 


\section{RESULTADOS Y DISCUSIÓN}

Procedimos al estudio cualitativo de todos los casos de oraciones (téticas y categóricas) en los que hubo diferencia en la ubicación de los sujetos en relación con el verbo entre las dos traducciones al español. El objetivo es analizar qué estructura informativa asignó a los enunciados cada traductor, por medio del análisis de su sintaxis.

Las oraciones téticas y categóricas que presentaron discrepancia sintáctica entre las dos traducciones al español fueron 13, divididas en tres grupos:

Grupo 1: Cambio en el orden sujeto-verbo entre las dos traducciones sin alteración de función sintáctica (7 casos)

Grupo 2: Cambio en el orden sujeto-verbo entre las dos traducciones con alteración de función sintáctica (3 casos)

Grupo 3: ajustes sintácticos para la introducción de referentes nuevos en el texto (3 casos).

\subsection{Grupo 1. Enunciados téticos con discrepancia de orden sujeto-verbo entre una y otra traducciones sin alteración de función sintáctica}

Los conjuntos de ejemplos (1) a (6) a continuación son casos de enunciados cuyo orden de palabras presentó discrepancia entre la primera traducción y la segunda. A continuación se presentan los conjuntos de ejemplos ${ }^{5}$.

(1a) RF: Eu trabalhava em um jornal popular como repórter de polícia.

(1b) AD:Trabajaba yo en un diario popular, como reportero de la sección Policiales.

(1c) JO:Yo trabajaba en un diario popular como reportero policial.

El ejemplo (1) es la primera oración del cuento. Constituye un enunciado tético de función introductiva, según Sasse (1996:33). Este autor (1996:32) distingue cinco tipos de oraciones téticas, según su función. Al ejemplo (1) le corresponde la función introductiva, al tratarse de una oración en la que todos los elementos son nuevos, al no haber texto previo, y tiene la función de introducir referentes que vendrán a formar una cadena tópica.

Así, en el texto original (1a), la primera posición de la sentencia (y del texto) viene ocupada por un referente totalmente nuevo. El orden es sujeto-verbo debido

5. En los ejemplos, los de la serie (a) corresponden al original (1975), (b) a la primera traducción (1978) y (c) a la segunda traducción (2014). 
a características de la gramaticalización de la relación sujeto-verbo en esa lengua. Se observa que en la primera traducción (1b) se decide ubicar el SN yo en posición posverbal, acorde con el carácter tético de dicho enunciado. Como se ha discutido, el orden SV en la primera referencia a determinado referente es esperado para una lengua que diferencia sintácticamente enunciados categóricos de téticos, como es el caso del español. Así, en (1b) ese SN nuevo, antes de que venga a constituir un tópico y punto de partida para una cadena tópica, entra en el texto en posición posverbal.

La segunda traducción (1c) subvierte el carácter tético claramente asignado al enunciado por la primera traductora. Recordando la cita de Wangi Cege (2013) de la introducción, aquí y en todos los ejemplos de sentencias téticas, ambos traductores al español tienen que poner de relieve una información que está indeterminada en el texto fuente por características de la lengua meta y para ello tienen que hacer elecciones sintácticas que tienen repercusiones informativas. Así, si en PB una tética puede presentar el orden SV y, con eso, por la pura sintaxis son indistinguibles de las categóricas, eso significa que el orden de palabras no diferencia enunciados téticos de categóricos en esa lengua. Cuando se traduce ese texto al español, en el que el orden sí distingue el valor informativo de los enunciados, los traductores se hacen responsables de asignar un valor informativo determinado al enunciado. En (1c), que presenta un ordenamiento distinto de (1b), el traductor elige ubicar el sujeto en posición preverbal. Al hacerlo, convierte un enunciado, que en la primera traducción había sido designado como tético, en categórico, pues el traductor, al ubicar el sujeto en posición preverbal, hace que este venga a ser interpretado como un tópico en cuyo entorno la proposición gravita. Una de las consecuencias interpretativas es que el sujeto-narrador arranca como centro de la narración y no es el caso, ya que el tema del cuento es la vida dentro de la redacción de un diario escrito para mujeres.

El mero hecho de que el sujeto aparezca en posición preverbal en el original (1a), a diferencia del español, no asigna a ese referente ningún estatus topical de antemano, una vez que la forma en que la relación sujeto-verbo se ha gramaticalizado en esa lengua hace que el sujeto aparezca en posición inicial, preverbal.

(2a) RF: Está tudo podre, no ponto, é só esperar.

(2b) AD:Está todo podrido, a punto. Sólo hay que saber esperar.

(2c) JO:Todo está podrido, sólo hay que esperar.

En (2) hay una oración tética de función descriptiva, siempre según la clasificación propuesta por Sasse (1996:35). Las téticas de función descriptiva, 
según este autor, se diferencian de las anteriores, de función presentativa, porque pueden aparecer en cualquier lugar del texto y tienen la función de presentar un comentario lateral con relativa independencia del desarrollo de los sucesos principales narrados. Así, presenta un estado de cosas en el que cualquier referente que aparezca, incluso si es sujeto, no es ni será la base de ninguna cadena tópica. La mayoría de los enunciados analizados en este artículo presentan esa función. En la primera traducción (2b) se emplea la colocación [está todo+adjetivo]. Este orden coincide con el de la colocación del original (2a). Ya en la segunda traducción (1c), se ubica el SN "todo" en posición preverbal. Tanto una como otra construcción son colocaciones legítimas en español y no se puede asignar el carácter marcado a ninguna de las dos. Los rasgos semánticos del sujeto todo no permiten que a este se le dé una interpretación específica, de manera que en (1c), aunque el orden es SV, sigue siendo un enunciado tético. Esto ya estaba previsto por Sasse (1987), que hace hincapié en que no se puede desprender la estructura informativa a partir de la mera disposición de los elementos en la oración.

(3a) RF: Ele estava tão irritado que não perguntei ao que Mulher se propunha.

(3b) $A D$ :Estaba tan irritado que no le pregunté qué se proponía Mujer.

(3c) JO:Estaba tan enojado que no pregunté lo que Mujer se proponía.

En (3a) se observa que la gramaticalización de la relación sujeto-verbo en $\mathrm{PB}$, lengua del original hace que incluso en el contexto de una oración relativa el orden sea SV. Eso plantea una serie de indefiniciones al traductor ya que la oración ao que Mulber se propunba tanto puede ser una tética, en que Mulber no es el centro en torno al cual gravita la proposición, como puede ser una categórica, que destaca Mulher como tópico, una vez que es un referente ya mencionado en el texto, y, por tanto, conocido en el discurso. La solución de la traductora (3b) es igualmente indeterminada. En español rioplatense y en otras variedades se observa una tendencia a ubicar en posición posverbal los sujetos de oraciones relativas y de algunas subordinadas. La indeterminación de (3b) estriba en si "Mujer" aparece en posición posverbal debido a una tendencia sintáctica o por una presión discursivopragmática, si la oración es tética. En (3c) el traductor quita la indefinición y asigna interpretación categórica al enunciado: "mujer" es un tópico, y aparece en posición preverbal en una construcción marcada, contrariando la tendencia sintáctica de ubicación posverbal de ese tipo de sujeto. Curiosamente, la distribución sintáctica entre el original y (3c) es la misma, pero informativamente no podrían ser enunciados más distintos entre sí. 
(4a) RF: Quantos livros você acha que Peçanha já leu?

(4b) AD: ¿Cuántos libros crees que leyó Peçanha?

(4c) JO: ¿Cuántos libros crees que Peçanha ha leído?

El conjunto (4) corresponde a otra oración relativa, ubicada dentro de una interrogativa. En (4a) el sujeto Peçanha es preverbal y la oración puede ser tanto tética como categórica. En (4b) al ubicar el sujeto en posición posverbal, la traductora indetermina, una vez más, si la oración es categórica - con el sujeto posverbal como resultado de una presión sintáctica - o tética, con el sujeto posverbal como resultado de una presión discursiva. En (4c) nuevamente el traductor acude a deshacer la indeterminación, y elige una construcción categórica, marcada.

(5a) RF: (...) empulhadores com seus computadores, todos criando a Grande Mentira.

(5b) $A D:(. .$.$) bromistas con sus computadoras, todos creando la Gran Mentira.$

(5c) JO: (...) estafadores con computadores, creando todos la Gran Mentira.

El ejemplo (5) corresponde a otra oración tética de función descriptiva, en la que uno de los personajes se desahoga con otro, criticando la sociedad. En este ejemplo, como en (2), el carácter semántico indeterminado de todos hace que el enunciado sea tético, tanto en el original como en (5b) o (5c), aunque el orden es distinto según la traducción.

(6a) RF: É isso mesmo que eu não quero.

(6b) $A D$ : Es eso mismo lo que no quiero.

(6c) $J O$ :Eso es justo lo que no quiero.

El conjunto en (6) presenta un enunciado categórico, una vez que, informativamente, se compone de dos elementos, un foco y una presuposición. El foco identificativo (É. KISS, 1998b) viene presentado por medio de una oración hendida en el original (6a) en la que el elemento enfocado, isso mesmo, aparece entre el verbo ser y la conjunción que. En la primera traducción (6b) se presenta la misma configuración sintáctica e informativa. En la segunda traducción (6c) el recurso empleado es otro procedimiento de escisión, una hendida inversa, que, sin embargo, preserva la estructura informativa binaria foco-presuposición del original.

(7a) RF: Quem tem que se defender é você mesma.

(7b) $A D:$ Tú misma eres quien tiene que defenderse.

(7c) JO:Quien se tiene que defender eres tú misma. 
En el conjunto (7) también se observa una oración categórica caracterizada por la presencia de la dicotomía informativa foco-presuposición. Esta vez, el foco identificativo se obtiene en el original (7a) por medio del recurso a la seudohendida. En la primera traducción (7b) se emplea el recurso a la seudohendida inversa, recurso asociado a la expresión de foco contrastivo. En la segunda traducción (7c) se observa el recurso a la seudohendida canónica presente en el original y evitado en la primera traducción. La estructura informativa binaria queda preservada en las dos traducciones.

\subsection{Grupo 2: Cambios de orden con alteración en la función sintáctica del sujeto}

Los conjuntos de ejemplos (8) a (10) a continuación son casos de enunciados téticos o categóricos cuyo orden de palabras presentó discrepancia entre la primera traducción y la segunda, identificados como téticos y cuya función sintáctica del sujeto cambia entre una traducción y otra.

(8a) RF: Um menino rico é roubado pelos ciganos e dado por morto.

(8b) AD:Un niño rico es robado por los gitanos y lo dan por muerto.

(8c) JO:A un chico rico lo roban unos gitanos y lo dan por muerto.

El ejemplo (8a) corresponde a una oración tética de función introductiva. El personaje es instado a crear una historia en el mismo momento y empieza a narrarla. Los referentes presentes en esa oración vendrán (o no) a convertirse en elementos de una cadena de referentes, pero es la primera vez que se los menciona en el texto. Tiene estructura pasiva sintáctica. La misma estructura es aprovechada en la primera traducción (8b), en la que se preserva la función sintáctica original, aunque la pasiva sintáctica en español rioplatense tiene una frecuencia mucho más baja que en PB en determinados registros (BARRENECHEA y MANACORDA, 1979, sobre el español rioplatense). En la segunda traducción (8c) se decide preservar la distribución preverbal del referente y a la vez se evita la pasiva sintáctica, menos frecuente en la lengua meta que en PB. La solución empleada fue la de convertir dicho referente en complemento directo y asignarle la función informativa tópico. La topicalización fuerza la división binaria entre tópico y comentario y la interpretación categórica a esa oración.

(9a) RF: Um dos ciganos é morto pela polícia.

(9b) AD:Uno de los gitanos es baleado por la policía.

(9c) JO:A uno de los gitanos lo mata la policía. 
El ejemplo (9a) corresponde, esta vez, a un enunciado categórico, pues la narración prosigue y los gitanos son ya un referente presentado previamente en el discurso. El sujeto acumula la función informativa tópico y encabeza una oración pasiva sintáctica, y difiere informativamente del ejemplo anterior porque la oración correspondiente era una tética de configuración sujeto-verbo. En el presente ejemplo, de configuración categórica, debido a las propiedades semánticas y pragmáticas de ese referente la interpretación también es categórica en la primera traducción (9b), que preserva, al igual que en el ejemplo anterior, la estructura del original. En la segunda traducción (9c) este elemento que ya tiene estatus topical se convierte en adjunto (correferente del complemento directo) y preserva la función informativa tópico. La función sintáctica cambia, pero la estructura informativa queda preservada en las dos traducciones.

(10a) RF: De mulher para mulher era assinada por uma tal Elisa Gabriela.

(10b) AD:De Mujer a Mujer era firmada por una tal Elisa Gabriela.

(10c) JO:De mujer a mujer lo firmaba una tal Elisa Gabriela.

En (10) sucede precisamente lo mismo que en el conjunto anterior de ejemplos. El sujeto de (10a) es el título de una sección del diario, ya mencionado previamente en el texto y, por lo tanto, al estar definido y ser conocido en el discurso, le corresponde la función pragmática tópico en una configuración categórica. En la primera traducción (10b), se preservan las estructuras sintáctica e informativa y en la segunda traducción (10c) el sujeto "De mujer a mujer", que ya tiene estatus topical se convierte sintácticamente en adjunto correferente del complemento directo y se preserva la función informativa tópico.

\subsection{Grupo 3: ajustes sintácticos para la introducción de referentes nuevos en el texto}

Los conjuntos de ejemplos (11) a (13) a continuación son casos de enunciados cuyo orden de palabras presentó discrepancia entre la primera traducción y la segunda, identificados como téticos y cuya función sintáctica del sujeto cambia entre una traducción y otra.

(11a) RF: Os charutos eram ordinários, estávamos no verão, de janelas fechadas, e o aparelho de ar-condicionado não funcionava bem.

(11b) $A D$ :Los habanos eran ordinarios, estábamos en verano, con las ventanas cerradas y el aparato de aire acondicionado que no funcionaba bien. 
(11c) JO:Los puros eran ordinarios, estábamos en verano, con las ventanas cerradas, y el aire acondicionado no funcionaba bien.

En (11a) el sujeto o aparelho de ar condicionado, mencionado por primera vez en el original aparece dentro de una oración tética descriptiva en la que el protagonista describe la situación después que se encienden unos puros dentro del despacho en el que se encuentra. Este SN es NUEVO, aunque inmediatamente se vincula a la descripción ambiental de un despacho. Sin embargo, la decisión de la traductora fue la de no presentarlo en posición inicial en forma de oración coordinada SV de apariencia categórica, sino de optar por una escisión: "Estábamos en verano (...) con (...) el aparato de aire acondicionado que no funcionaba bien". Se trata de un procedimiento claramente destopicalizador, en línea con la propuesta de Lambrecht (2000) discutida en la sección (1). Con eso, logra cambiar la función sintáctica del sujeto $o$ aparelho de ar condicionado del original quitándole cualquier rasgo topical. Esa operación recuerda al clásico ejemplo de Lambrecht (1994) de una oración de foco sentencial en francés, en respuesta a preguntas del tipo "¿qué pasó?":

J'ai ma voiture qui est en panne

Yo-tengo mi auto que está en avería

"Mi auto tiene un problema"

En el ejemplo el SN ma voiture al ser NUEVO y de baja presuposicionalidad no puede encabezar una oración. Primero tiene que ser introducido en el discurso con función sintáctica de complemento, en posición posverbal y luego recuperado como sujeto/tópico elidido de la oración relativa encabezada por "que". El procedimiento adoptado en la traducción es el mismo, en atención a las restricciones del español respecto a la ubicación en primera posición de un referente de estatus informativo NUEVO. El traductor, en (11c) a su vez, decide ubicar el referente como sujeto en la oración coordinada tal cual el original, asignándole lo que buscaba evitar la primera traductora, una predicatividad sintáctica, es decir, una apariencia categórica a una oración tética. Este no es un procedimiento atípico en la prosa de ficción publicada en Chile, como lo han notado Soto y Castro (2007). En su estudio, aunque predominan los referentes DADOS en el discurso en posición preverbal, aparece una minoría de referentes NUEVOS en esa posición, como estrategia destinada a lograr ciertos efectos interpretativos, como el de forzar en el lector familiaridad con determinado referente, aunque este es nuevo. 
(12a) RF: Já tive outro consulente que, como você, também gostava de se vestir de mulher.

(12b) AD:Tuve ya otro consultante al que le gustaba vestirse de mujer.

(12c) JO:A otro seguidor como tú también le gustaba vestirse de mujer.

El ejemplo (12a) es de una tética, en la que el personaje, autor de una sección en el diario, hace un comentario lateral en la respuesta a una carta de un lector. En la primera traducción (12b) se decide mantener el carácter tético, que asigna cierta irrelevancia a lo que se dice, sin relieve a ninguno de los referentes. En la segunda traducción (12c) se asigna relieve al complemento, que mantiene la función sintáctica pero informativamente pasa a tópico y, por lo tanto, gana una interpretación de elemento más central en la narrativa que sigue. Con eso, además la oración pasa a categórica, y se diferencia tanto de la interpretación tética del original como de la primera traducción.

(13a) RF: Éramos nós dois, eu e Maria.

(13b) AD:Eso éramos nosotros dos, yo y María.

(13c) JO:Éramos los dos, María y yo.

El ejemplo (13a) corresponde a la respuesta que determinado lector del diario escribe en una carta. Dicha oración aparece después de la pregunta retórica "¿Sabes lo que es que dos personas se gusten?" La primera traducción (13b) presenta la solución de añadir un pronombre demostrativo como sujeto, como forma de adecuar la expresión a los límites de la sintaxis de la lengua. Aunque en la lengua del original Éramos nós dois, eu e Maria sea sintácticamente una respuesta perfectamente aceptable a la pregunta formulada inmediatamente antes, la traductora decide por no presentar ese sintagma como respuesta, e introduce el demostrativo "eso" con función reasuntiva en respuesta al carácter abierto de la pregunta. En la segunda traducción (13c) no se observa ninguna alteración estructural en la respuesta en relación con el original, más allá de la elección por el orden no marcado en español entre los sintagmas "yo" y "María".

\section{CONCLUSIÓN}

El análisis contrastivo cualitativo de las dos traducciones de Corazones Solitarios publicadas en Sudamérica ha revelado algunos recursos discrepantes empleados por los autores en lo que se refiere a la interacción entre pragmática y sintaxis en la traducción de oraciones téticas y categóricas del portugués al español. 
Los resultados muestran recursos que preservan la estructura informativa y otros que la cambian. Hubo cambios en la función sintáctica de referentes en los que se veía un esfuerzo por preservar la estructura informativa del original y alteraciones en la estructura sintáctica entre el original y la traducción con el objetivo de introducir referentes nuevos en cuyas soluciones se pudo observar los límites de la sintaxis de una lengua frente a otra y la desigual relación entre pragmática y sintaxis entre las dos lenguas.

Las discrepancias señaladas en las soluciones sintácticas elegidas por los distintos traductores muestra, por un lado, la riqueza de las posibilidades expresivas del español, pero, por otro, alteraciones significativas en la forma de interpretar dichos enunciados que, muchas veces, cambian la interpretación de lo que se dice.

\section{REFERENCIAS BIBLIOGRÁFICAS}

BARRENECHEA, A.M.; MANACORDA DE ROSETTI, M.V. (1979). La voz pasiva en el español hablado en Buenos Aires. In: Barrenechea, A.M. et alli (eds.). Estudios linguïsticos y dialectológicos. Buenos Aires: Hachette.

É. KISS, K. (1998b). Identificational Focus versus Information Focus. Language 74, pp. 245273.

FONSECA, R. (1975).Corações Solitários. In: Feliz Ano Novo. Rio de Janeiro: Artenova.

FONSECA, R. (1977). Corazones solitarios. Trad. Pablo del Barco. In: Feliz año nuevo. Madrid: Alfaguara.

FONSECA, R. (1978). Corazones solitarios. Trad. Andrea Diessler. In: Quince cuentistas brasileños de hoy. Buenos Aires: Sudamericana.

FONSECA, R. (2014). Corazones solitarios. Trad. John O’Kuinghttons. In: Feliz año nuevo. Santiago: Tajamar Editores.

GUTIÉRREZ ORDÓÑEZ, S. (1997).Temas, remas, focos, tópicos y comentarios, Madrid: Arco Libros.

HAJICOVÁ, E.; SGALL, P. (2003). Information structure, translation and discourse. In: Gerzymisch-Arbogast, H. et al. (eds.). Textologie und Translation. Tübingen: G. Narr. 
HASSLER, G. (2008), Tema, rema, focos y tópicos en la oralidad fingida y en su traducción, In: Brumme, J.; Resinger, H. (eds.). La oralidad fingida: obras literarias: descripción y traducción, Iberoamericana/Vervuert: Frankfurt am Main/Madrid.

KATO, M. (1989). Tópico e sujeto: duas categorias na sintaxe? Cadernos de Estudos Linguísticos 17, pp. 109-131.

LAMBRECHT, K. (1994), Information structure and sentence form: Topic, focus, and the mental representation of discourse referents, Cambridge: Cambridge University Press.

LAMBRECHT, K. (2000). "When subjects behave like objects: An analysis of the merging of $\mathrm{S}$ and $\mathrm{O}$ in sentence-focus constructions across languages". Studies in Language 24, pp. 611-82.

PINHEIRO-CORREA, P. (2015). Características das construções de foco sentencial no português, em um estudo de corpus paralelo. ReVEL, edição especial n. 10, 195-222.

SASSE, H.J. (1987). "The thetic/categorical distinction revisited", Linguistics 25, pp. 511-80.

SASSE, H.J. (1996). Theticity. Arbeitspapier 27 (Neue Folge), Universität zu Köln, Institut für Sprachwissenschaft.

SASSE, H.J. (2006). Theticity. In Bernini, G.; Schwarz, M.L. (eds.). Pragmatic organization of discourse in the languages of Europe. Berlin: Mouton de Gruyter.

SORNICOLA, R. (1995). Theticity, VS order and the interplay of syntax, semantics and pragmatics.SpracbtypologieundUniversalienforscbung, 48.

SOTO, G.; CASTRO, C. (2007). Sobre los sintagmas nominales preverbales en el español oral y escrito en Chile: gramática y discurso. Boletín de Filología XLII, pp. 341-367.

WANGUI CEGE, D. (2013). Topic and focus entities in German and English utterances: mismatches and translation challenges. Dissertação de Mestrado, University of Nairobi.

ZUBIZARRETA, M. L. (1999). Las funciones informativas: tema y foco. In: Bosque, I; Demonte, V. (eds.). Gramática descriptiva de la lengua española. Madrid: Espasa, pp. 4215 44.

Recebido: 10/02/2018

Aceito: 25/02/2018 